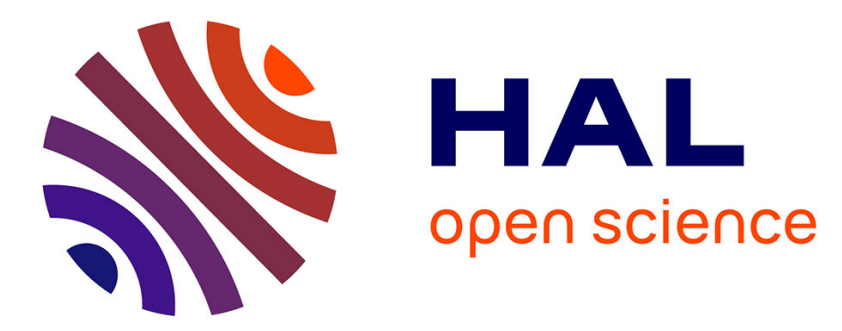

\title{
A Comparative Analysis of Pulsating Carrier Injection vs. Extended Kalman Filter Injection-Based Sensorless Control
}

Andreea Beciu, Emmanuel Godoy, Pedro Rodriguez-Ayerbe, Maalouf Amira, Imen Bahri

\section{To cite this version:}

Andreea Beciu, Emmanuel Godoy, Pedro Rodriguez-Ayerbe, Maalouf Amira, Imen Bahri. A Comparative Analysis of Pulsating Carrier Injection vs. Extended Kalman Filter Injection-Based Sensorless Control. IEEE Vehicle Power and Propulsion Conference (VPPC), Dec 2017, Belfort, France. pp.1-5, 10.1109/vppc.2017.8331017 . hal-01813827

\section{HAL Id: hal-01813827 https://hal.science/hal-01813827}

Submitted on 10 Apr 2020

HAL is a multi-disciplinary open access archive for the deposit and dissemination of scientific research documents, whether they are published or not. The documents may come from teaching and research institutions in France or abroad, or from public or private research centers.
L'archive ouverte pluridisciplinaire HAL, est destinée au dépôt et à la diffusion de documents scientifiques de niveau recherche, publiés ou non, émanant des établissements d'enseignement et de recherche français ou étrangers, des laboratoires publics ou privés. 


\title{
A Comparative Analysis of Pulsating Carrier Injection vs. Extended Kalman Filter Injection-Based Sensorless Control
}

\author{
Andreea-Livia Beciu 1),2), Emmanuel Godoy ${ }^{1)}$, Pedro Rodriguez-Ayerbe ${ }^{1)}$, Amira Maalouf ${ }^{2)}$ and Imen Bahri ${ }^{3)}$ \\ 1) CentraleSupélec, L2S, CNRS, Université Paris-Saclay, Gif-sur-Yvette, France \\ 2) Thales-AES, 41 Bvd. de la République, 78400, Chatou, France \\ 3) GEEPS - Group of electrical engineering, Paris, Gif-sur-Yvette, France \\ Email: Andreea.Beciu@centralesupelec.fr
}

\begin{abstract}
Sensorless control for electrical drives is still a challenge in the low-speed and standstill region. Solutions that inject a high frequency signal in order to generate positiondependent harmonics are well known for their good performance in this particular speed range. The possibility to combine signalinjection techniques with model-based observers could be a solution for sensorless control over the whole speed range.

In this paper, a pulsating vector carrier signal injection is used with two different estimation methods. First method is based on the commonly used filtering and demodulation associated to the pulsating carrier method, and for the second method, an injection-based Extended Kalman Filter is proposed in order to estimate the rotor position. This two methods are analysed, we concentrate on: parameter tuning, transitory response and noise attenuation. The analysis is supported by experimental measurements obtained on a salient-pole permanent magnet synchronous machine. The results show the satisfactory performance of both methods and the potential to use the proposed injection-based Extended Kalman Filter from standstill to the high-speed region.
\end{abstract}

\section{INTRODUCTION}

Due to their high efficiency and power density, synchronous machines are now widely employed in propulsion applications in different sectors, from automotive to aeronautics, for example, in the context of the more electric aircraft. For torque, speed or position control of this drives, precise knowledge of the rotor position is required. However, the suppression of the associated mechanical position sensor is motivated by multiple reasons: increase of cost, weight or integration problems. The difficulty to estimate the position is that, under no excitation, at standstill or very low speed, the system is not observable.

To surpass the standstill observability problem, high frequency (HF) signal injection (HFSI) methods to estimate the rotor position have been proposed during the last two decades [1]-[5]. In the case of a permanent magnet synchronous machine (PMSM), this methods rely on spatial magnetic saliencies of the machine that provide position-dependent harmonics. Nevertheless, these methods are suitable on a limited speed range (standstill and low-speed); for higher speeds, it is common to use a transition algorithm [6], [7] in order to switch the estimation source to a high speed dedicated algorithm, based on back-EMF estimation, like the Extended Kalman Filter (EKF).

In the context of embedded applications, using only one algorithm over the whole speed range is a very attractive solution. In the literature we find some applications that follow this approach - for PMSM in [9] or for a SynRM machine, in [10], a simple method is proposed - to use a single algorithm to merge the saliency information from the signal injection with the fundamental information from the back-EMF of the machine: the Extended Kalman Filter, a model basedtechnique, potentially providing position estimation over the entire speed-range. Furthermore, a similar approach is used in the case of a non-salient machine: a High Frequency (HF) signal is injected in the estimated $d$ - axis, meant to make the rotor vibrate. The model, taking into account the known injection vector, becomes observable and the EKF algorithm can be used in order to estimate the rotor position, see [11].

In this paper we will propose a similar approach for a salient machine, to take into account the signal injection vector and design an injection-based EKF with the new model. This method will be then compared to its saliency-tracking HFSI counterpart. The high frequency excitation chosen for both methods is the pulsating carrier voltage signal injection on the estimated $d$-axis of the rotor reference frame. The advantage of this injection method is that when the position is well estimated, a minimum current ripple is induced on the $q-$ axis, the axis producing the torque [1]. Using the same excitation for both methods, we would like to analyse the accuracy and the advantages of using one method or the other.

The paper is organised as follows: section II presents the equations of a salient PMSM in the rotating frame with some observability remarks and the choice of the injection signals; using this injection signals, section III, presents the principle and the demodulation scheme for pulsating HFSI and then, the extended Kalman Filter approach. Finally, section IV presents the experimental test bench and the key comparative aspects with the corresponding experimental results. Finally, conclusions are summarized in section $\mathrm{V}$. 


\section{Model of The PM SYNChronous MACHINE}

The mathematical model, in the rotor reference frame, for a salient-type PMSM is presented in eq. (1). The following notations are used: $v_{d}, v_{q}, i_{d}$ and $i_{q}$ are respectively the stator voltages and currents, $\omega_{e}$ and $\theta$ are the rotor electrical speed and position respectively, $R_{s}$ the stator phase resistance, $L_{d}, L_{q}$ the direct and quadrature inductances respectively and $\psi_{f}$ the permanent flux of the magnets.

$$
\begin{aligned}
& v_{d}=R_{s} i_{d}+\frac{d \psi_{d}}{d t}-\omega_{e} \psi_{q} \\
& v_{q}=R_{s} i_{q}+\frac{d \psi_{q}}{d t}+\omega_{e} \psi_{d}
\end{aligned} \text { with } \begin{aligned}
& \psi_{d}=L_{d} i_{d}+\psi_{f} \\
& \psi_{q}=L_{q} i_{q}
\end{aligned}
$$

The mechanical equation is presented in (2) with: $p$, the number of pole pairs, $\Gamma_{e}$, the electromagnetic torque, $\Gamma_{L}$ the load torque, $J$, the total moment of inertia and $f$, the viscous friction coefficient.

$$
J \frac{d \omega_{e}}{d t}+f \omega_{e}=p\left(\Gamma_{e}-\Gamma_{L}\right), \quad \Gamma_{e}=\frac{3}{2} p\left(\psi_{d} i_{q}-\psi_{q} i_{d}\right) .
$$

The observability for electrical drives based on the mathematical model has been thoroughly addressed in recent research and precise observability conditions have been synthesised for the electric machines [9], [12]. Using the notation $\Delta L=L_{d}-L_{q}$, the observability analysis, based on the rank criterion approach, reveals the following form for the determinant of the observability matrix [9]:

$$
\Delta_{\omega_{e}=0, \dot{\omega}_{e}=0}=\frac{\Delta L}{L_{d} L_{q}}\left(\frac{d i_{q}}{d t}\left(i_{d} \Delta L+\psi_{f}\right)-i_{q} \Delta L \frac{d i_{d}}{d t}\right)
$$

It can be observed that the local observability condition is guaranteed in presence of a measurable saliency $(\Delta L \neq 0)$ and when the derivatives of the currents $i_{d}$ and $i_{q}$ are different from zero. Based on this considerations, high frequency injection signals are considered for standstill at low speed operation [5].

Nevertheless, the excitation signals are known to the user. When model-based techniques of estimation are employed, it is possible to take into account the expression of the injection signals in the motor model, as a known disturbance. For example, the extended-model in the stationary frame proposed in [11], considering the injection signals in the model, shows that under HF signal injection (eq. (4)), a non-salient PMSM, can be observable at standstill when the estimation error is different from zero.

Let us consider a high frequency signal superimposed on the estimated d-axis command of the stator eq. (4), at a known carrier frequency $\left(\omega_{h}\right)$ and amplitude $\left(V_{h}\right) \cdot v_{\hat{d}}^{h}$ and $v_{\hat{q}}^{h}$ in (4) stand for the injected stator voltages in the rotor estimated reference frame.

$$
v_{\hat{d}}^{h}=V_{h} \cos \left(\omega_{h} t\right), v_{\hat{q}}^{h}=0 .
$$

This high frequency signal is also known as a pulsating carrier signal injection [1], [2]. Assuming there is a position estimation error, $\hat{\theta}=\theta+\Delta \theta$, the injected tensions in the stator reference frame will depend on this error:

$$
\left|\begin{array}{c}
v_{\alpha}^{h} \\
v_{\beta}^{h}
\end{array}\right|=V_{h}\left|\begin{array}{c}
\cos (\theta+\Delta \theta) \\
\sin (\theta+\Delta \theta)
\end{array}\right| \cos \left(\omega_{h} t\right) .
$$

\section{DERIVED POSITION ESTIMATORS}

\section{A. Saliency tracking for pulsating carrier injection}

The current response to the injection signal is obtained using a high frequency model of the machine [1]. The measured current contains the stator frequency fundamental component at $I_{1}$ amplitude, and the induced high frequency currents $\left(\tilde{i}_{\alpha \beta}\right)$ :

$$
i_{\alpha \beta_{\text {meas }}}=I_{1} e^{j\left(\omega_{e} t\right)}+\tilde{i}_{\alpha \beta} .
$$

In the presence of a magnetic saliency, the resulting HF currents in the $\alpha \beta$ reference frame contain harmonics with information of the real position $\theta$. For simplicity reasons, we present in eq. (7) the four high frequency harmonics in $\alpha \beta$ reference frame [2], [4]:

$$
\begin{aligned}
\tilde{i}_{\alpha \beta}= & I_{p} e^{j\left(\omega_{h} t+\hat{\theta}-\frac{\pi}{2}\right)}+I_{n} e^{j\left(-\omega_{h} t+2 \theta-\hat{\theta}+\frac{\pi}{2}\right)} \\
& +I_{p} e^{j\left(-\omega_{h} t+\hat{\theta}+\frac{\pi}{2}\right)}+I_{n} e^{j\left(\omega_{h} t+2 \theta-\hat{\theta}-\frac{\pi}{2}\right)}
\end{aligned}
$$

Using the notations $l_{\Sigma}=\frac{L_{d}+L_{q}}{2}$ and $l_{\Delta}=\frac{L_{d}-L_{q}}{2}$, the expression of the harmonics amplitudes is given by:

$$
I_{p}=\frac{V_{h}}{2 \omega_{h}} \frac{l_{\Sigma}}{L_{q} L_{d}} \text { and } I_{n}=\frac{V_{h}}{2 \omega_{h}} \frac{l_{\Delta}}{L_{q} L_{d}} .
$$

To facilitate the demodulation, the currents are transposed to $d q$ reference frame in eq. (8) and reduced to a simple form in eq. (9).

$$
\begin{aligned}
\tilde{i}_{d q}= & I_{p} e^{j\left(\omega_{h} t-\frac{\pi}{2}\right)}+I_{n} e^{j\left(-\omega_{h} t-2 \Delta \theta+\frac{\pi}{2}\right)} \\
& +I_{p} e^{j\left(-\omega_{h} t+\frac{\pi}{2}\right)}+I_{n} e^{j\left(\omega_{h} t-2 \Delta \theta-\frac{\pi}{2}\right)} \\
\tilde{i}_{d q}= & \left(I_{p}+I_{n} e^{-j 2 \Delta \theta}\right) e^{j\left(\omega_{h} t-\frac{\pi}{2}\right)} \\
& +\left(I_{p}+I_{n} e^{-j 2 \Delta \theta}\right) e^{j\left(-\omega_{h} t+\frac{\pi}{2}\right)} \\
\tilde{i}_{d q}= & 2\left(I_{p}+I_{n} e^{-j 2 \Delta \theta}\right) \sin \left(\omega_{h} t\right)
\end{aligned}
$$

Eq. (9) can be represented under a matrix form:

$$
\left|\begin{array}{c}
\tilde{i}_{d} \\
\tilde{i}_{q}
\end{array}\right|=2 I_{p}\left|\begin{array}{c}
\sin \left(\omega_{h} t\right) \\
0
\end{array}\right|+2 I_{n}\left|\begin{array}{c}
\cos (2(\theta-\hat{\theta})) \\
\sin (2(\theta-\hat{\theta}))
\end{array}\right| \sin \left(\omega_{h} t\right)
$$

From eq. (10) it can be seen that the HF component of $i_{q}$ is direct proportional to the estimation error:

$$
\tilde{i}_{q}=2 I_{n} \sin (2(\theta-\hat{\theta})) \sin \left(\omega_{h} t\right) \approx 4 I_{n}(\theta-\hat{\theta}) \sin \left(\omega_{h} t\right)
$$

The interest in this kind of injection is to act on a single flux direction, in our case, the considered injection is on the $d$ - estimated axis. We see in eq. (10) that when there is no estimation error, $\Delta \theta=0$, the HF current on the $q$-axis will become zero. Therefore, a minimum couple ripple is assured as long as the rotor position is well estimated.

Figure 1 illustrates the common spatial saliency demodulation process using pulsating HFSI. By minimising $\Delta \theta$, the estimation error is forced to zero. A tracking observer based on the mechanical system model is used in order to achieve a zero-lag estimation [1]. 
Parameter choice: The tuning of the pulsating carrier method lies in the observer characteristics but also on the lowpass filter.

Higher the frequency of injection signal, better the decoupling between the HF signals and the fundamental command of the machine. However, there is a limitation on the injection frequency that is related to the inverter's capacities. In the experimental setup, because of the low value of the frequency of the injection signal, the high-pass and the low-pass filter were set with a cut off frequency of $50 \mathrm{~Hz}, 10 \%$ of the injection frequency. The observer is then tuned as a trade-off between accuracy and noise-level. The bandpass of the closed loop, containing the low-pass filter and the observer is set to $140 \mathrm{~Hz}$.

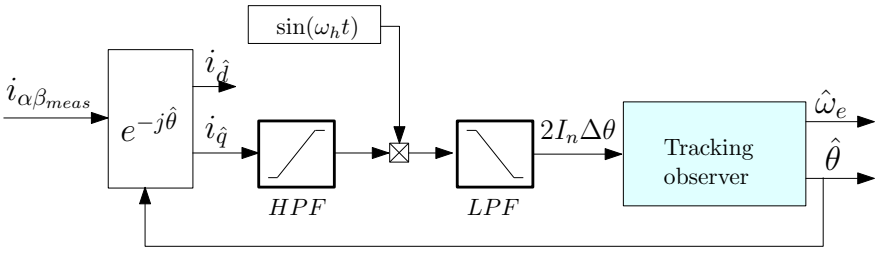

Fig. 1: Demodulation of the measured stator currents $\left(i_{\alpha \beta_{\text {meas }}}\right)$ for pulsating HFSI position estimation

\section{B. Extended Kalman Filter approach}

Using the same injection signals, a discrete Extended Kalman Filter can be designed to perform position and velocity estimation. The interest of using an Extended Kalman Filter is its good performance over a wide range of speed. By employing the proposed HFSI in eq. (4), the bandwidth can be extended to low speed and standstill as well.

There is more than one model that can be used for position estimation with an EKF. The $d-q$ model, in rotor reference frame, is usually employed for high speed operation (see [13]), due to its precision and reduced number of operations for a salient motor. Figure 2 shows experimental results for low speed operation of an EKF under no-load, in presence of HFSI, described in eq (4). The mechanical equation is taken into account. However, experience has shown important stationary error at standstill and low-speed.

The model in stationary frame has one major advantage of being able to take into account the injection signal into the model and the prediction step of the EKF algorithm [11].

The mathematical model for a salient PMSM in stationary frame requires high computation time due to its complexity. If we admit an incertitude over the motor's inductances, one can suppose $L_{d}=L_{q}=L_{s}$, which leads to an EKF using the non-salient model of the synchronous machine [6], [8], and thus reduce the complexity. This model is further presented in eq. (12-14) with the following notations: $v_{\alpha}, v_{\beta}, i_{\alpha}$ and $i_{\beta}$ are respectively the stator voltages and currents, $v_{\alpha}^{*}$ and $v_{\beta}^{*}$ are the fundamental part of the command of the machine, while $v_{\alpha}^{h}$ and $v_{\beta}^{h}$ are the HF injection signals.

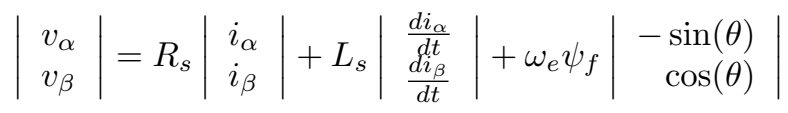

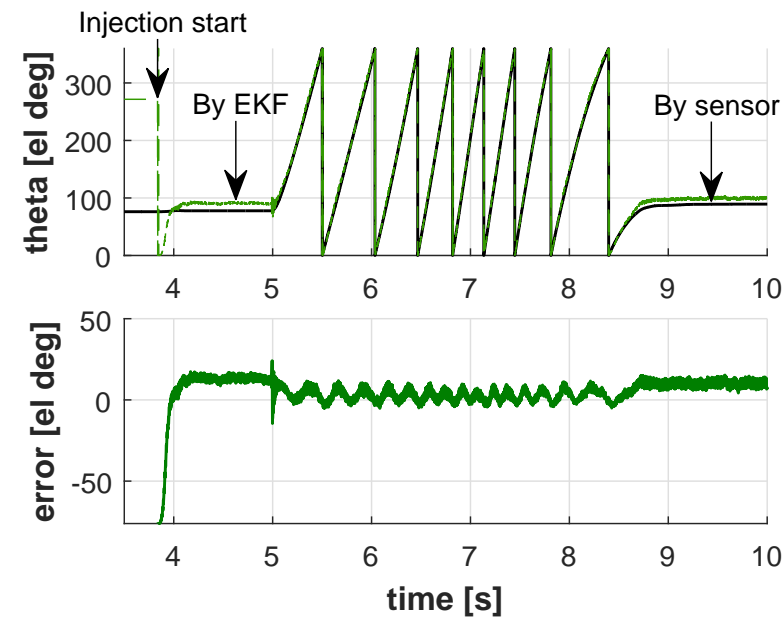

Fig. 2: Experimental results for EKF using the $d-q$ model with pulsating HFSI in speed closed loop: standstill and low speed operation (from standstill to 100rpm)

$$
\left|\begin{array}{c}
v_{\alpha} \\
v_{\beta}
\end{array}\right|=\left|\begin{array}{c}
v_{\alpha}^{*} \\
v_{\beta}^{*}
\end{array}\right|+\left|\begin{array}{c}
v_{\alpha}^{h} \\
v_{\beta}^{h}
\end{array}\right|
$$

The stationary frame electromagnetic torque for a nonsalient machine:

$$
\Gamma_{e}=\frac{3}{2} p\left(i_{\beta} \cos (\theta)-i_{\alpha} \sin (\theta)\right) \Psi_{f}
$$

The non-linear state-space model of the non-salient PMSM can be resumed, using the already-mentioned equations, under the following form:

$$
\left\{\begin{array}{l}
\dot{x}=f(x, u) \\
y=C x
\end{array}\right.
$$

With:

$$
x=\left[\begin{array}{lllll}
i_{\alpha} & i_{\beta} & \theta & \omega_{e} & \Gamma_{L}
\end{array}\right], u=\left[\begin{array}{llll}
v_{\alpha}^{*} & v_{\beta}^{*} & v_{\alpha}^{h} & v_{\beta}^{h}
\end{array}\right] \text { and } y=\left[\begin{array}{ll}
i_{\alpha} & i_{\beta}
\end{array}\right] \text {. }
$$

The resisting torque is part of the state vector and it is considered slow varying, $\frac{d \Gamma_{L}}{d t}=0$. The non-linear dynamics can be written in the following form:

$$
f(x, u)=\left(\begin{array}{c}
\frac{1}{L_{s}}\left(v_{\alpha}^{*}+v_{\alpha}^{h}-R_{s} i_{\alpha}+\omega_{e} \Psi_{f} \sin (\theta)\right) \\
\frac{1}{L_{s}}\left(v_{\beta}^{*}+v_{\beta}^{h}-R_{s} i_{\beta}-\omega_{e} \Psi_{f} \cos (\theta)\right) \\
\omega_{e} \\
\frac{1}{J}\left(p\left(\Gamma_{e}-\Gamma_{L}\right)-f \omega_{e}\right) \\
0
\end{array}\right)
$$

The key point is that $\frac{d u}{d x} \neq 0$, because the injection signals in eq. (5) are dependent on the estimated position. As a result, position derivatives of the injection signals will be contained in the Jacobian of the machine model, $F_{k-1}$ in (17).

The continuous-time system presented in eq. (15) is discretized with a first-order Euler approximation. Consider the new discrete time non-linear system:

$$
\left\{\begin{array}{l}
x_{k+1}=f\left(x_{k}, u_{k}\right)+w_{k}, \quad k=0,1,2 . . \\
y_{k}=h\left(x_{k}\right)+v_{k}
\end{array}\right.
$$


Where $f$ is a vector of non-linear functions that describe the system dynamics and $h$ is the observation equation. We note with $x_{k}$, the vector of state variables, $u_{k}$ the command vector, $y_{k}$ the output vector, $w_{k}$ and $v_{k}$ the process and measurement noise respectively.

The Extended Kalman Filter algorithm has a recursive structure, similar to that of the Linear Kalman Filter:

- The initialisation phase, at $k=1$ :

$$
\hat{x}_{0 \mid 0}=x_{0}, P_{0 \mid 0}=P_{0}
$$

- The prediction step, between $k-1$ and $k$, the model of the machine is first linearised at the beginning of the sampling period around the current state, then $P_{k \mid k-1}$ is computed:

$$
\begin{gathered}
F_{k-1}=\left.\frac{\partial f(x, u)}{\partial x}\right|_{\hat{x}_{k-1 \mid k-1}, u_{k-1}} \\
\hat{x}_{k \mid k-1}=f\left(\hat{x}_{k-1 \mid k-1}, u_{k-1}\right) \\
P_{k \mid k-1}=F_{k-1} P_{k-1 \mid k-1} F_{k \mid k-1}^{T}+Q
\end{gathered}
$$

- The update step for moment $k$ :

$$
\begin{aligned}
& \hat{x}_{k \mid k}=\hat{x}_{k \mid k-1}+K_{k}\left(y_{k}-h\left(\hat{x}_{k \mid k-1}\right)\right) \\
& K_{k}=P_{k \mid k-1} C_{k}^{T}\left[C_{k} P_{k \mid k-1} C_{k}^{T}+R\right]^{-1} \\
& P_{k \mid k}=P_{k \mid k-1}-K_{k} C_{k} P_{k \mid k-1}
\end{aligned}
$$

With:

$$
C_{k-1}=\left.\frac{\partial h(x)}{\partial x}\right|_{\hat{x}_{k \mid k}}
$$

1) Parameter choice: The difficulty of the EKF algorithm lies in the tuning of the covariance matrices. The covariance matrix of the prediction error, $P_{0}$, is initialized according to the accuracy of the initial guess $\left(x_{0}\right)$. The covariance matrix of the observation noise, $R$, and the covariance matrix of the process noise, $Q$, were chosen by trial and error method:

$$
\begin{array}{r}
\operatorname{diag}\left(P_{0}\right)=\left[\begin{array}{lllll}
1 & 1 & 1 & 1 & 1
\end{array}\right], \\
\operatorname{diag}(R)=\left[\begin{array}{llll}
15 & 15
\end{array}\right], \operatorname{diag}(Q)=\left[\begin{array}{lllll}
1 & 1 & 10^{-4} & 10^{-4} & 2
\end{array}\right] .
\end{array}
$$

Comparing to methods that demodulate the injection signal like pulsating HFSI, EKF needs more memory and computational time. Nonetheless, modern computational devices are capable of running high performance algorithms with a low execution time. A comparison of DSP and FPGA solutions for sensorless speed controller for a synchronous machine using EKF is presented in [14].

\section{EXPERIMENTAL VALIDATION}

\section{A. Description of the experimental bench}

The proposed algorithms are tested on a $4.8 \mathrm{~kW}$ salient PMSM and the real position is provided by a 1024-pulse incremental encoder, used only in order to validate the estimation results. A $10 \mathrm{~kW}$ commercial inverter is used with $10 \mathrm{kHz}$ frequency of the PWM and $2 \mu s$ dead-time. The characteristics of the used PMSM are given in Table I.

The real-time control is modelled in MATLAB/Simulink and realised using dSpace MicroLabBox development system.

\section{TABLE I: MOTOR PARAMETERS}

\begin{tabular}{|c|c|}
\hline Rated power & $4.8 \mathrm{~kW}$ \\
\hline Number of pole pairs $(\mathrm{p})$ & 2 \\
\hline Stator phase resistance $\left(R_{s}\right)$ & $0.86 \Omega$ \\
\hline Direct inductance $\left(L_{d}\right)$ & $17 \mathrm{mH}$ \\
\hline Quadratic inductance $\left(L_{q}\right)$ & $41 \mathrm{mH}$ \\
\hline Rotor flux $\left(\psi_{f}\right)$ & $0.14 \mathrm{~Wb}$ \\
\hline Total moment of Inertia $(J)$ & $0.0023 \mathrm{~kg} . \mathrm{m}^{2}$ \\
\hline
\end{tabular}

The sampling period for estimation and control loop is fixed to $0.1 \mathrm{~ms}$. The pulsating carrier injection signal has $V_{c}=15 \mathrm{~V}$ amplitude and $500 \mathrm{~Hz}$ frequency.

\section{B. Experimental results}

1) Standstill operation: In Figure 3 we can see the convergence phase for both observers at standstill, after the injection starts, no-load operation. Algorithms are initialised with 60 electrical degrees of error. For EKF, the convergence time is dependent on the injection amplitude $V_{h}$. If we double the amplitude of the injection signal, $V_{h}=30 \mathrm{~V}$, the convergence time is reduced to $0.05 \mathrm{~s}$. The amplitude could be eventually real-time adjusted in an adaptive way to assure a fast convergence time and it could also be stopped as the speed increases and the electromotive-force becomes high enough, see [9].

Transitory response: The sensorless operation with speed control, under no load is shown in figures 4 and 5 . The speed reference goes from 100rad/s to $-100 \mathrm{rad} / \mathrm{s}$. We can observe an acceptable estimation error of \pm 10 electrical degrees for both methods. Pulsating HFSI is not influenced by the speed or acceleration, but it is highly influenced by noise and unmodeled secondary saliencies that produce a position-dependent error.

The amplitude of the estimation error is reduced in the case of the injection-based EKF estimation, however an acceleration dependency can be observed as a bias on the estimation error. This error can be explained by the use of a non-salient model in the EKF algorithm. Improvement of the model is part of an ongoing-study.
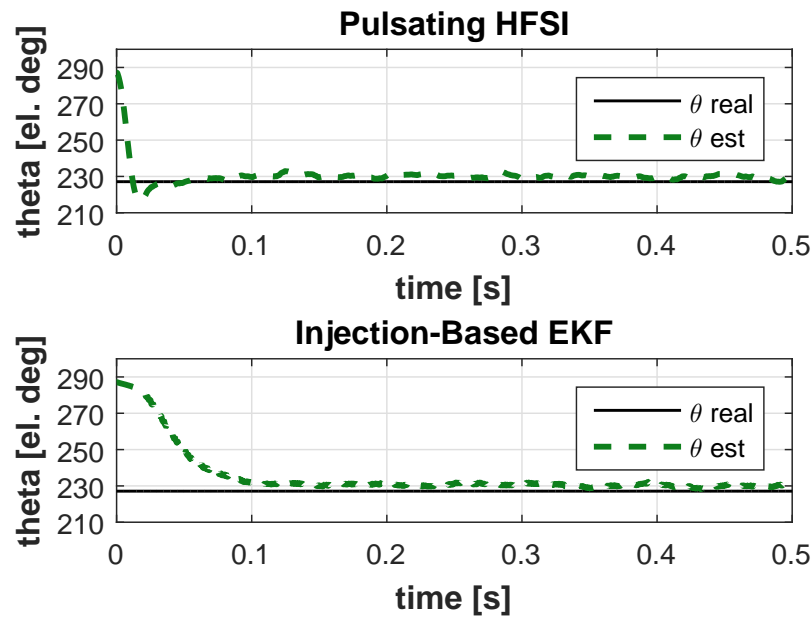

Fig. 3: Experimental results for standstill operation 

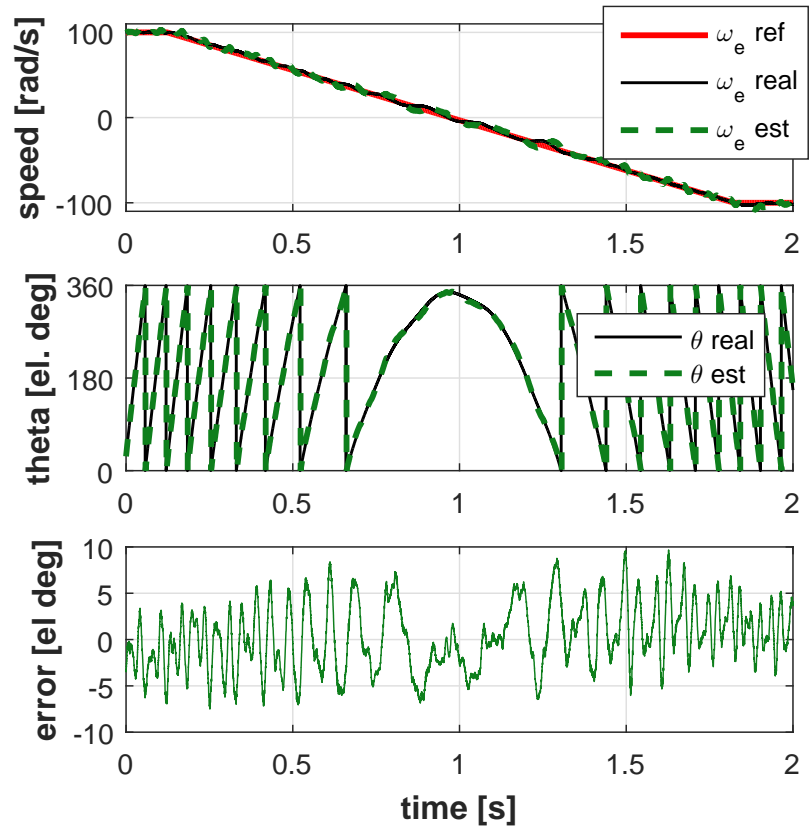

Fig. 4: Experimental results for demodulation with pulsating carrier injection in speed closed loop
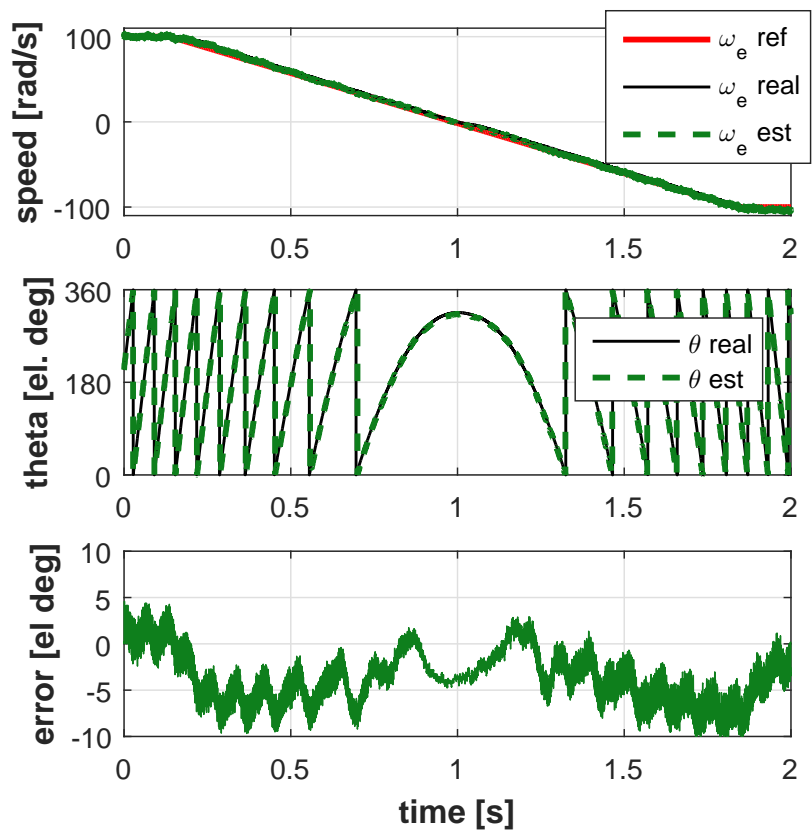

Fig. 5: Experimental results for EKF in stationary frame with pulsating carrier injection in speed closed loop

\section{CONClusion}

This paper has presented two different approaches for position estimation that are employing a pulsating carrier injection. The EKF is generally employed for high speed estimation of the position. It is shown that by taking into account the injection signals, EKF with the non-salient model in stationary frame provides satisfactory estimation results compared to the classical pulsating HFSI position estimation. As a result, EKF's bandwidth could be extended to low speed and standstill as well.

The experimental results show that EKF seems to be less sensitive to measurement noise and secondary saliencies, compared to the pulsating HFSI. However, EKF is affected by the accuracy of the model and the incertitude on the model parameters. Future work will be focused on developing an injection-based EKF using an improved model in the stationary frame, taking into account the injection signal. As the pulsating demodulation methods are sensitive to cross-inductance and saturation, it is interesting to see how is the injection-based Kalman Filter affected by this factors.

\section{REFERENCES}

[1] M. J. Corley and R. D. Lorenz, "Rotor position and velocity estimation for a salient-pole permanent magnet synchronous machine at standstill and high speeds," in IEEE Transactions on Industry Applications, vol. 34, no. 4, pp. 784-789, Jul/Aug 1998.

[2] D. Raca, P. Garcia, D. Reigosa, F. Briz and R. Lorenz, "A comparative analysis of pulsating vs. rotating vector carrier signal injection-based sensorless control," Applied Power Electronics Conference and Exposition, 2008.

[3] F. Briz and M. W. Degner, "Rotor Position Estimation," in IEEE Industrial Electronics Magazine, vol. 5, no. 2, pp. 24-36, June 2011.

[4] D. D. Reigosa, P. Garcia, F. Briz, D. Raca and R. D. Lorenz, "Modeling and Adaptive Decoupling of High-Frequency Resistance and Temperature Effects in Carrier-Based Sensorless Control of PM Synchronous Machines," in IEEE Transactions on Industry Applications, vol. 46, no. 1, pp. 139-149, Jan.-feb. 2010.

[5] P. Garcia, F. Briz, M. W. Degner and D. Diaz-Reigosa, ”Accuracy and Bandwidth Limits of Carrier Signal Injection-Based Sensorless Control Methods," IEEE Industry Applications Conference Forty-First IAS Annual Meeting, Tampa, FL, pp. 897-904, 2006.

[6] H. Than, Y. S. Kung and L. C. Huang, "Digital hardware implementation of a sensorless speed controller for PMSM drives using HF signal injection and EKF," 2016 International Conference on Advanced Materials for Science and Engineering (ICAMSE), Tainan, pp. 192-195, 2016.

[7] A. Rambetius and B. Piepenbreier, "Sensorless Control of Wound Field Synchronous Machines for the Whole Speed Range," Proceedings of PCIM Europe, Nuremberg, Germany, 2015, pp. 1-8.

[8] S. Bolognani, L. Tubiana and M. Zigliotto, "Extended Kalman filter tuning in sensorless PMSM drives," in IEEE Transactions on Industry Applications, vol. 39, no. 6, pp. 1741-1747, Nov.-Dec. 2003.

[9] M. Koteich, A. Maloum, G. Duc and G. Sandou, "Observability analysis of sensorless synchronous machine drives," Control Conference (ECC), European, Linz, pp. 3560-3565, 2015.

[10] R. Caro, C. A. Silva, R. Prez and J. I. Yuz, "Sensorless control of a SynRM for the whole speed range based on a nonlinear observability analysis," 2017 IEEE International Conference on Industrial Technology (ICIT), Toronto, ON, Canada, pp. 336-341, 2017.

[11] F. Abry, A. Zgorski, X. Lin-Shi and J-M. Retif, "Sensorless position control for SPMSM at zero speed and acceleration," in Proc. 2011 European Conference on Power Electronics and Applications, pp. 1-9, 2011.

[12] D. Zaltni, M. N. Abdelkrim, M. Ghanes and J. P. Barbot, ”Observability analysis of PMSM," 2009 3rd International Conference on Signals, Circuits and Systems (SCS), Medenine, pp. 1-6, 2009.

[13] A. Maalouf, S. Le Ballois, L. Idkhajine, E. Monmasson, J. Y. Midy and F. Biais, "Sensorless control of brushless exciter synchronous starter generator using Extended Kalman Filter," 2009 35th Annual Conference of IEEE Industrial Electronics, Porto, 2009, pp. 2581-2586.

[14] L. Idkhajine, E. Monmasson and A. Maalouf, "Fully FPGA-Based Sensorless Control for Synchronous AC Drive Using an Extended Kalman Filter,' in IEEE Transactions on Industrial Electronics, vol. 59, no. 10 , pp. 3908-3918, Oct. 2012 